\title{
Simultaneously measuring ocular aberration and anterior segment biometry during accommodation
}

\author{
Yuanyuan Wang, Yilei Shao and Yimin Yuan* \\ School of Optometry and Ophthalmology and Eye Hospital \\ Wenzhou Medical University, Wenzhou 325027 \\ Zhejiang Province, P. R. China \\ *yuanyimin1226@hotmail.com
}

Received 17 March 2014

Accepted 3 May 2014

Published 3 June 2014

\begin{abstract}
In the human eye, accommodation is essential for functional vision. However, the mechanisms regulating accommodation and the ocular parameters affecting aberrations remain to be explored. In order to measure the alterations of ocular aberration and crystalline lens biometry during dynamic accommodative stimuli, we designed an optical coherence tomography with ultra-long penetration depth (UL-OCT) combined with a Shack-Hartmann wavefront sensor (SHWFS). This integrated set up measures human eye's anterior segment as well as monochromatic high-order aberrations (HOAs) with $6 \mu \mathrm{m}$ resolution and $(1 / 20) \lambda$ accuracy. A total of 10 healthy volunteers without ocular diseases were examined. Upon exposure to accommodative stimuli, the wavefront aberrations became larger. Among the anterior segment biometry, the anterior crystalline lens demonstrated significant curvature during accommodation and was the major cause of high-order aberration. These findings suggest that the front surface of the crystalline lens can significantly affect variation among aberrations, which is a key factor underlying the quality of human vision.
\end{abstract}

Keywords: Optical coherence tomography; wavefront sensor; accommodation.

\section{Introduction}

Accommodation is the process by which the human eye changes its optical power to form a clear image on retina with changing distance. Previous work by Helmholtz and Schachar has shown that altering the curvature of both anterior surfaces and posterior surfaces of crystalline lenses plays a critical role in dynamic accommodation. ${ }^{1,2}$ Crystalline lenses continue to grow throughout life, and their shape and structure undergo significant changes with age. ${ }^{3}$ In order to identify the mechanisms underlying accommodation and age-related hyperopia, it is necessary to study the biometric changes of the crystalline lens in response to dynamic stimuli. ${ }^{4}$

Many imaging techniques have been developed and used in order to examine crystalline lenses in vivo. ${ }^{5-7}$ optical coherence tomography (OCT) can perform rapid, noncontact, cross-sectional, high

This is an Open Access article published by World Scientific Publishing Company. It is distributed under the terms of the Creative Commons Attribution 3.0 (CC-BY) License. Further distribution of this work is permitted, provided the original work is properly cited. 
resolution imaging that is widely used for examining the anterior segment. ${ }^{8,9}$ Near-infrared light sources can be used during image capture, which allows the eye to maintain its original accommodative positioning. This ability to capture ocular images without altering accommodation makes OCT highly advantageous for research in vivo.

The purpose of accommodation is to form a clear image on retina, but this process is not perfect. Unwanted wavefront aberrations, including defocus, coma, astigmatism, spherical aberrations, and some high-order aberrations (HOA), may become apparent during accommodation. Shack-Hartmann wavefront sensors (SHWFS) have been widely used in the measurement of ocular aberrations because of their high accuracy and ability to produce results in real time. ${ }^{10}$ Investigating the effects of ocular aberrations on accommodation is important for understanding how the human eye regulates the quality of images produced. Since crystalline lenses are the primary part of the eye that changes in response to focusing, it is believed that ocular aberration and crystalline lens biometry during dynamic accommodation has high correlation. ${ }^{9}$

Recently, we have succeeded in imaging the entire crystalline lens and measuring its biometry using spectral domain OCT with ultra-long penetration depth (UL-OCT). ${ }^{11}$ In order to evaluate anterior segment biometry and ocular aberrations simultaneously, we have combined UL-OCT with a customized SHWFS. ${ }^{12}$ In this study, we measured alterations in ocular aberrations and crystalline lens biometry and investigated their relationship during dynamic accommodation.

\section{Experimental Design}

Figure 1 shows the integrated UL-OCT and SHWFS system. The UL-OCT is based on the common spectral domain OCT schematic. This system achieves $6.0 \mu \mathrm{m}$ axial resolution by using $840 \mathrm{~nm}$ center wavelength and $50 \mathrm{~nm}$ bandwidth super luminescent diode (SLD). The total exposure power for OCT imaging is $1.30 \mathrm{~mW}$ which is safety for human eye. The cross-sectional image with $7.8 \mathrm{~mm}$ depth can be achieved by the spectrometer that consists of a grating (1800 lines/mm) and a line scans CCD (2048pixels). A full-range (equivalent depth $15.6 \mathrm{~mm}$ ) OCT image was obtained using this custom method that eliminated mirror artifacts of the raw image ${ }^{13}$ and allowed the whole human anterior segment to be imaged successfully. A SHWFS with a $32 \times 32$ microlens array was used to measure wavefront aberrations during accommodation. Light was emitted from a $780 \mathrm{~nm}$ laser diode (LD), entered the tested eye, was reflected by the retina, and passed through the microlens array to form a spot array on a charge-coupled device. The wavefront aberrations were reconstructed by calculating the

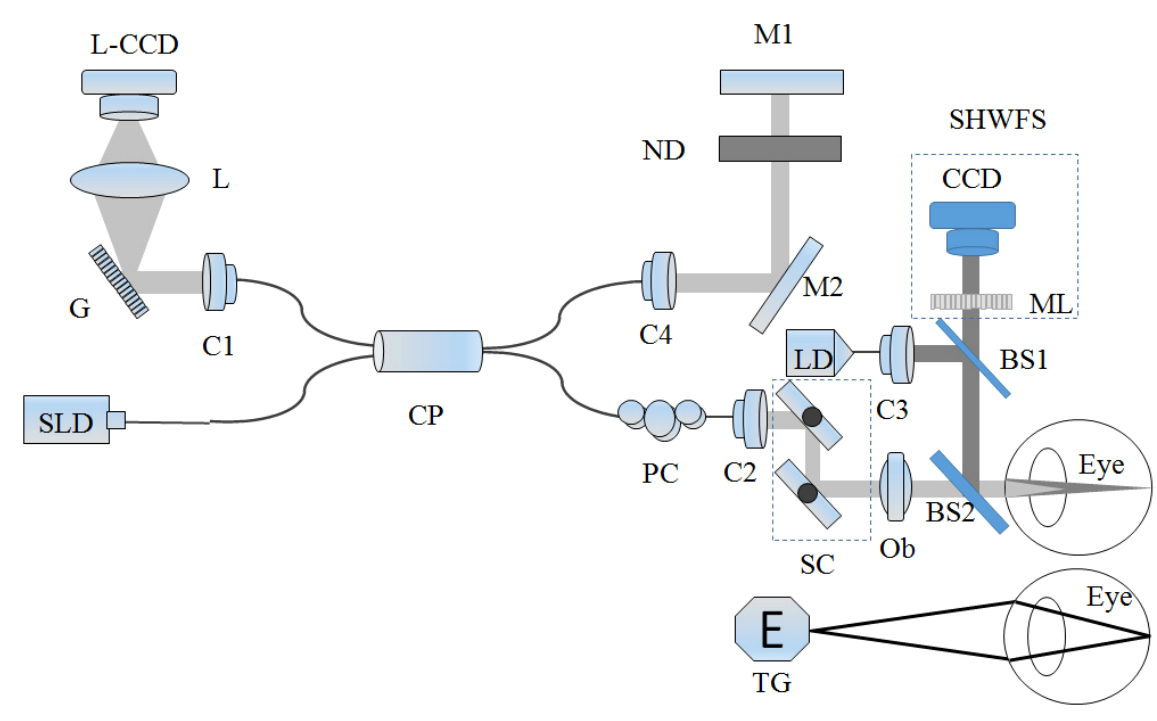

Fig. 1. Diagram of the integrated UL-OCT and SHWFS; SLD: super luminescent diode; CP: fiber coupler; C1 C4: fiber collimator; G: grating; L-CCD: line scan charge-coupled device; L: lens; ND: neutral density filter; M1, M2: mirror; PC: polarization controller; SC: X-Y scanner; Ob: objective; BS1, BS2: beam splitter; LD: laser diode; ML: micro-lens array; CCD:CCD camera; SHWFS: Shack-Hartmann wavefront sensor; TG: target. 
differences between the deformed and reference images. The SHWFS measured up to 35th-order Zernike aberrations in $20 \mathrm{~Hz}$ to an accuracy of $(1 / 20) \lambda$.

The UL-OCT and SHWFS were combined on a beam splitter in front of the tested eye. This allowed synchronous imaging and measurement of aberrations. The fixation target (TG) was a liquid-crystal display (LCD) showing a white letter "E" (size: 20/ 50) on black background. The test was performed in a darkened room to prevent artifacts produced by outside light. The TG was moved back and forth in front of the left eye to stimulate accommodation, and measurements were performed on the right eye. No mydriatics were administered, and defocus and astigmatism of the left eye were corrected by trail lenses. First, the TG was positioned $4.0 \mathrm{~m}$ (0D) in front of the trail lens before the left eye. After the subject visualized the letter "E" clearly, the anterior segment of the right eye was imaged using ULOCT, and ocular aberrations were recorded with SHWFS. After the first measurement, the TG was moved to a distance of $0.3 \mathrm{~m}(3 \mathrm{D})$ in front of the trail lens, which is the typical distance for reading.
Then the anterior segment and ocular aberration were measured for a second time.

Previous work has established a custom algorithm that corrects for the refraction at the anterior corneal and lens surfaces. ${ }^{10,13}$ Figure 2(a) shows the anterior segment image taken using this algorithm. Using UL-OCT imaging, we measured dimensional parameters, including anterior chamber depth (ACD), lens thickness (LT), and pupil diameter (PD). The anterior surface curvature (ASC) and posterior surface curvature (PSC) of lens were also obtained by calculating their radius of curvature. Wavefront aberrations were recorded at a frame rate of $20 \mathrm{~Hz}$ by the SHWFS. Each measurement was made an average of 20 times to eliminate random error. Ocular aberrations were recorded on the natural PD and were described in Zernike polynomials, as recommended by the Vision Science and Its Application (VISIA) Standards Taskforce team. The peak to valley value (PV) and root-meansquare (RMS) of HOA were calculated using Zernike coefficients of 6 th to 35 th order. Figures 2(c) and $2(d)$ show the wavefront of one volunteer's HOA before and after accommodation.

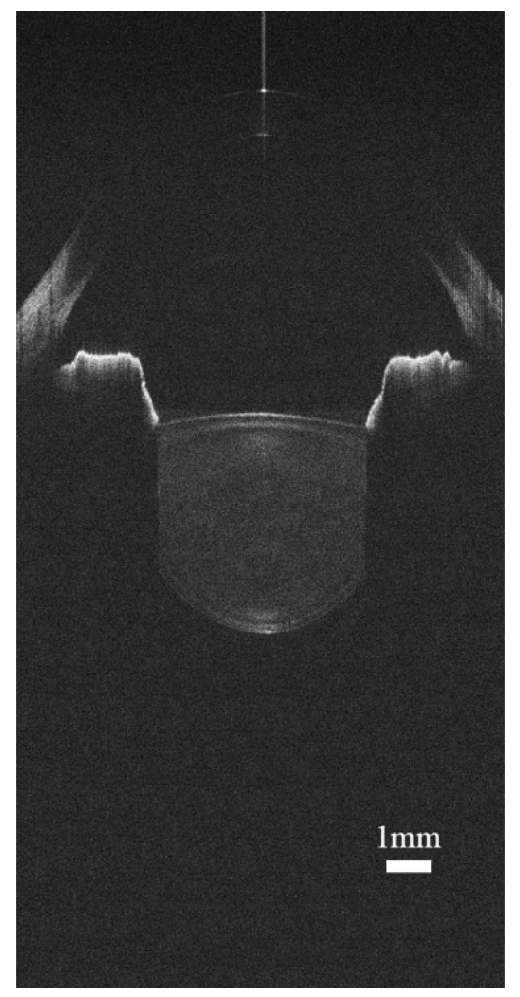

(a)

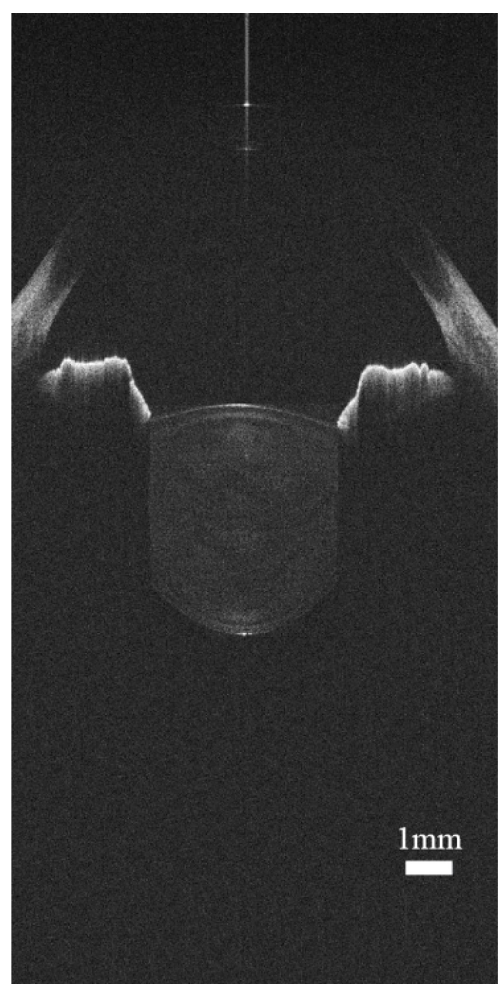

(b)

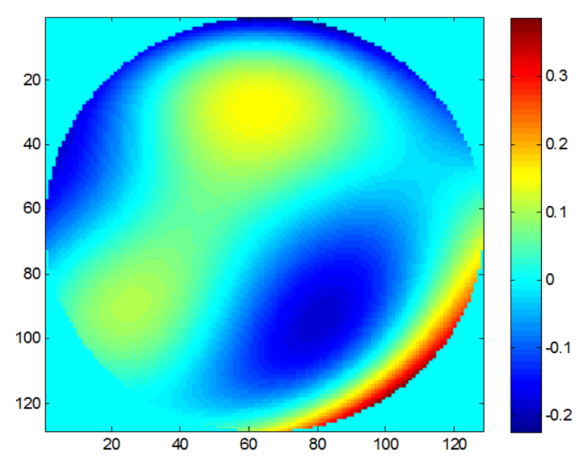

(c)

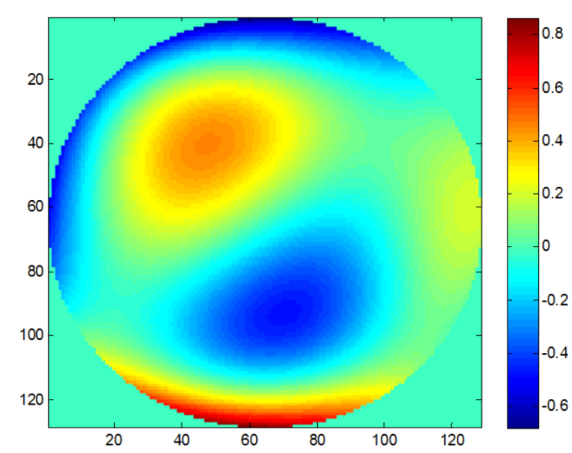

(d)

Fig. 2. (a) and (b) are the OCT images of anterior segments obtained for baseline and 3D accommodation. (c) and (d) are the wavefront under baseline and 3D accommodation conditions. 


\section{Results and Discussion}

This research was approved by the ethics committee of the School of Ophthalmology and Optometry at Wenzhou Medical University. And the process was conducted according to the principles of the Declaration of Helsinki. A total of 10 volunteers were recruited from among the students in the present laboratory, and each participant signed an informed consent form. There were 6 men $(60 \%)$ and $4(40 \%)$ women, aged from 20 to 31 years old $(24.32 \pm 3.32$; mean \pm s.d.). Each participant underwent a complete ophthalmic examination to confirm the lack of eye diseases, including myopia.

To eliminate accommodation artifacts from ambient light, the ocular measurements were made in a dark room. Following baseline measurements, the TG was moved from a distance of $4 \mathrm{~m}$ to $0.33 \mathrm{~cm}$ from the left eye of the participant, and the biometry of anterior segment and wavefront aberrations were recorded again under this second accommodative condition.

Figure 3 demonstrates the changes in wavefront aberrations of the 10 volunteers. Accommodation narrows the pupil, which suggests that when the diameter of the pupil is decreased, the high-order wavefront is reduced. However, our measurements demonstrated that after accommodation, aberrations in high-order wavefront became more numerous (Fig. 3). The mean accommodated HOA-PV of 10 volunteers was $1.639 \mu \mathrm{m}$, which is 2.82 times greater than that of a relaxed HOA-PV. Similarly, the mean accommodated HOA-RMS of the 10 volunteers was $0.263 \mu \mathrm{m}$ which was 3.13 times greater than baseline values. The purpose of accommodation is to focus images of near objects on the retina. Changes in lower order wavefront aberrations, particularly defocusing, are useful for vision. However, large HOAs have a significant effect on vision quality. This phenomenon is only caused by anterior segment altering.

Figure 4 shows the changes in biometric dimensions with accommodative status. After accommodative stimuli, the PD decreased in size, but the ACD only diminished slightly. The average rates of shrinkage were small: $15.5 \%$ and $3.3 \%$, for the PD and ACD, respectively. Similarly, the ACS was significantly reduced (average shrinkage of $20.3 \%$ ). These changes in ocular dimensions lead to slight increases in LT and PSC of $2.8 \%$ and $6.3 \%$, respectively.

The experimental results recorded by this system showed the decreases in PD and ACS to be the most pronounced following changes in accommodative stimuli, but the rest of the parameters demonstrated little change. As focal length decreased, the PD became smaller. Pupil size is inversely proportional to the wavefront aberrations. This would suggest that a smaller PD would cause reduced PV and RMS of HOAs. However, our measurements show the opposite results (Fig. 4). Our results suggest that as the focal length decreases, the ciliary muscles of the eye curve the anterior surface of the lens sharply to maintain image focus. This bending of the anterior surface of the lens also leads to rapid increases in the PV and RMS, which become larger than baseline values. These results indicate that changes in the ACS might be critical for focal length and that its sharpness also affects the variation in high-order aberrations that diminish vision quality.

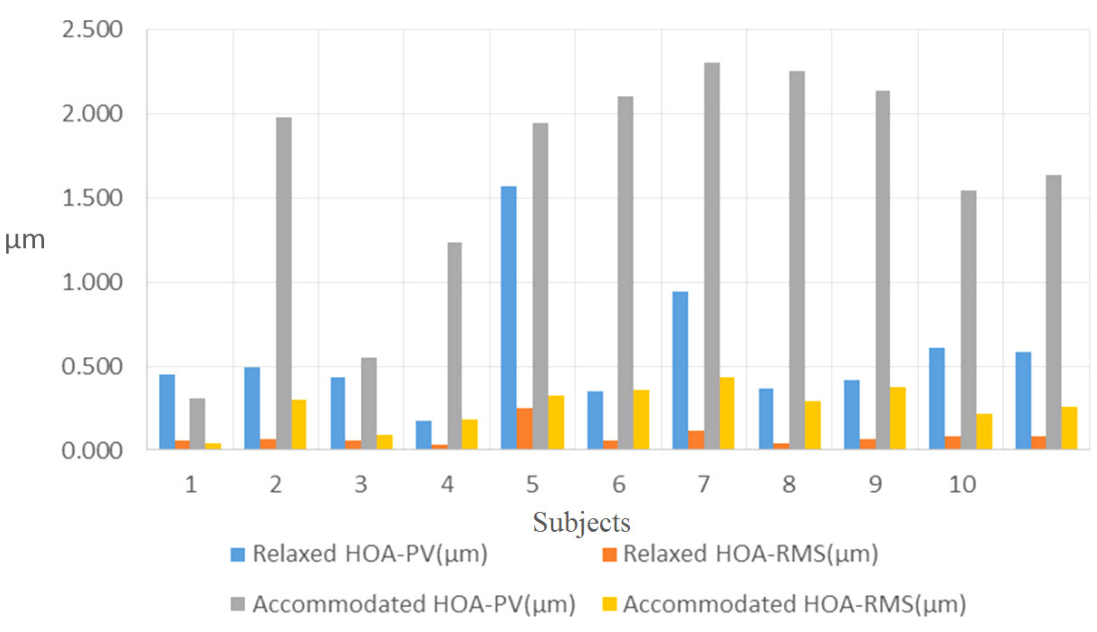

Fig. 3. Changes in 10 volunteers' high-order wavefront aberrations. 


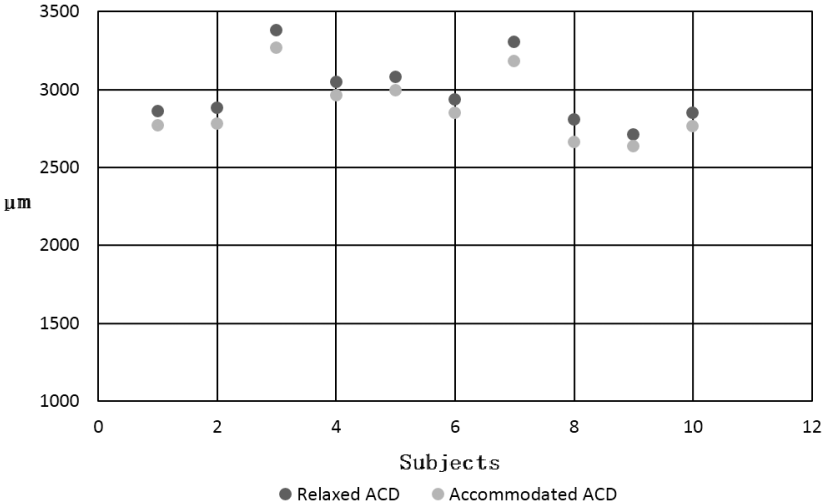

(a)

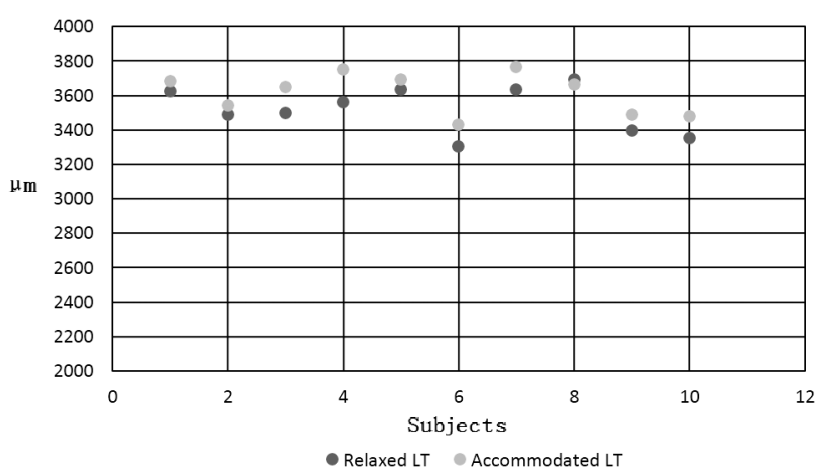

(c)

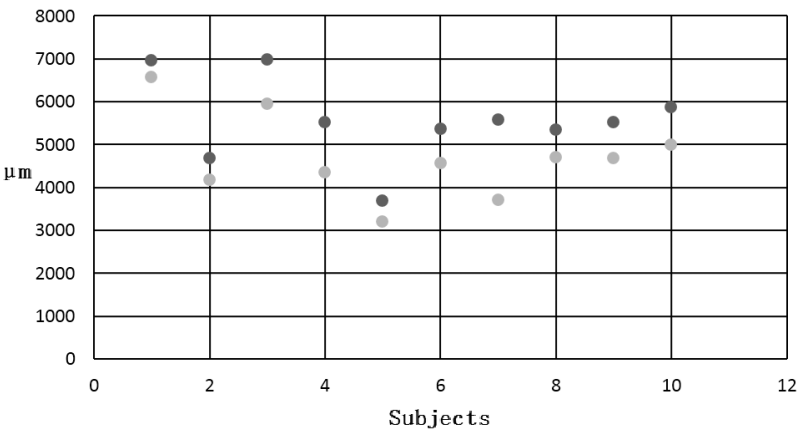

Relaxed PD Accommodated PD

(b)

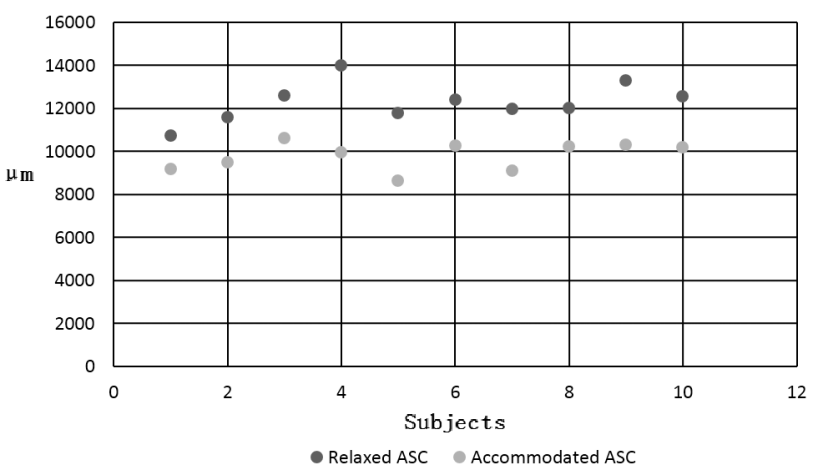

(d)

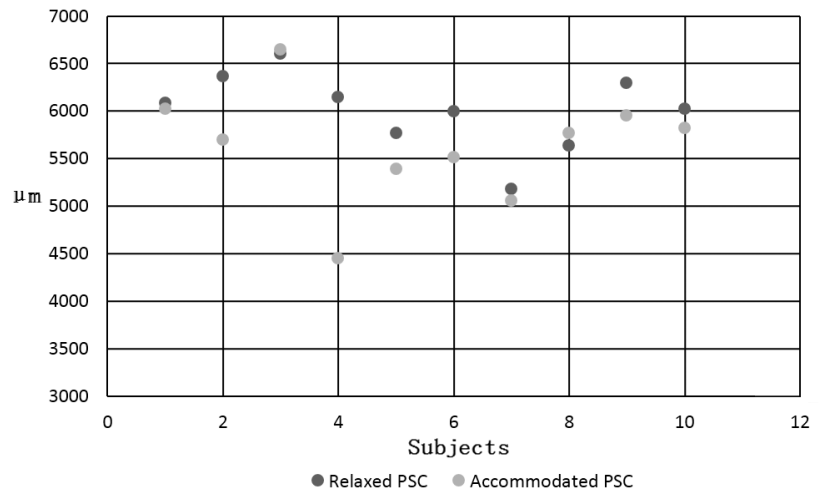

(e)

Fig. 4. Changes in biometric dimensions. ACD: anterior chamber depth; PD: pupil diameter; LT: lens thickness; ASC: crystalline lens's anterior surface curvature; PSC: crystalline lens's posterior surface curvature.

The data presented in this work is limited to 10 subjects. In order to perform statistical analysis with clinical significance, a large number of volunteers should be included. In future studies to investigate the mechanisms of accommodation, we will increase the number of volunteers to measure ACS and high-order ocular aberrations in order to attain a complete statistical analysis.

\section{Conclusion}

In conclusion, we combined the ultra-long penetration depth OCT with the SHWFS to one set up. The whole human eye's anterior segment was imaged and high order ocular aberrations were obtained through accommodative process in real time. Through movement of the vision target, we determined that the curve of the anterior surface of the 
lens changed during accommodation. This may be the major cause of the high-order aberrations. In the future, this device may be a powerful tool for analyzing the relationship between crystalline lens's anterior surface and high-order aberrations. The study provides a novel method to investigate the accommodative mechanism.

\section{Acknowledgments}

We acknowledge the support from the Zhejiang Province Science and Technology Development (Grant No. 2013C33170), the Zhejiang Provincial Natural Science Foundation of China (Grant No. Y1110383), and the Wenzhou Science and Technology Development (Grant No. Y20100174).

\section{References}

1. H. von Helmholtz, Ueber das Sehen des Menschen ein Populär Wissenschaftlicher Vortrag, Leopold Voss (1855) (in German).

2. R. A. Schachar, D. Cudmore, T. Black, "Experimental support for Schachar's hypothesis of accommodation," Ann. Ophthalmol. 25(11), 404-409 (1993).

3. M. Dubbelman et al., "Changes in the internal structure of the human crystalline lens with age and accommodation," Vis. Res. 43(22), 2363-2375 (2003).
4. L. F. Garner, M. K. Yap, "Changes in ocular dimensions and refraction with accommodation," Ophthalmic Physiol. Opt. 17(1), 12-17 (1997).

5. Y. Deng et al., "Numerical study of the effects of scatterer sizes and distributions on multiple backscattered intensity patterns of polarized light," Opt. Lett. 33(1), 77-79 (2008).

6. N. Tian et al., "Fluorescence ghost imaging with pseudothermal light," Opt. Lett. 36(16), 3302-3304 (2011).

7. W. Hu et al., "Nonlinear optical microscopy for histology of fresh normal and cancerous pancreatic tissues," PloS One 7(5), e37962 (2012).

8. I. Grulkowski et al., "Three-dimensional biometric measurements of accommodation using full-eyelength swept-source OCT," Invest. Ophtalmol. Vis. Sci. 54(6), 381 (2013).

9. C. de Freitas et al., "In vivo measurement of the average refractive index of the human crystalline lens using optical coherence tomography," Opt. Lett. 38(2), 85-87 (2013).

10. B. Grimm, S. Goelz, J. F. Bille, "Objective measurement of wave aberrations of the human eye with the use of a Hartmann-Shack wave-front sensor," JOSA A 11(7), 1949-1957 (1994).

11. Y. Yuan et al., "Repeated measurements of the anterior segment during accommodation using long scan depth optical coherence tomography," Eye 83 Contact Lens 38(2), 102 (2012).

12. G. Shi et al., "Measurement of ocular anterior segment dimension and wavefront aberration simultaneously during accommodation," J. Biomed. Opt. 17(12), 120501 (2012). 